\title{
Shift-Life Interactive Art: Mixed-Reality Artificial Ecosystem Simulation
}

\author{
Eugene Ch'ng ${ }^{\mathrm{a}}$, Dew Harrison ${ }^{\mathrm{b}}$ and Samantha Moore ${ }^{\mathrm{b}}$ \\ ${ }^{a}$ NVIDIA Joint-Lab on Mixed Reality, University of Nottingham Ningbo China, 199 Taikang East Road, \\ Zhejiang Ningbo China 315100. eugene.chng@nottingham.edu.cn \\ ${ }^{b}$ Faculty of Arts, University of Wolverhampton, Wulfruna Street, Wolverhampton WV1 1SB, United Kingdom. \\ dew.harrison@wlv.ac.ukands.moore@wlv.ac.uk
}

\begin{abstract}
This article presents a detailed design, development and implementation of a Mixed Reality Art-Science collaboration project which was exhibited during Darwin's bicentenary exhibition at Shrewsbury, England. As an artist-led project the concerns of the artist were paramount, and this article presents Shift-Life as part of an on-going exploration into the parallels between the non-linear human thinking process and computation using semantic association to link items into ideas, and ideas into holistic concepts. Our art explores perceptions and states of mind as we move our attention between the simulated world of the computer and the real-world we inhabit, which means that any viewer engagement is participatory rather than passive. From a Mixed Reality point of view, the lead author intends to explore the convergence of the physical and virtual, therefore the formalization of the Mixed Reality system, focusing on the integration of artificial life, ecology, physical sensors and participant interaction through an interface of physical props. It is common for digital media artists to allow viewers to activate a work either through a computer screen via direct keyboard or mouse manipulation, or through immersive means to activate their work, for "Shift-Life" the artist was concerned with a direct "relational" approach where viewers would intuitively engage with the installation's everyday objects, and with each other, to fully experience the piece. The Mixed Reality system is mediated via physical environmental sensors, which affect the virtual environment and autonomous agents, which in turn reacts and is expressed as virtual pixels projected onto a physical surface. The tangible hands-on interface proved to be instinctive, attractive and informative on many levels, delivering a good example of collaboration between the Arts and Science.
\end{abstract}

Keywords: mixed reality, artificial life, agent-based modeling, art, participatory, sensors

\section{Introduction}

The Mixed Reality installation titled "Shift-Life" presented in this article was exhibited at the Shift-Time Festival of Ideas (3-12 July 2009) which coincides with Charles Darwin's 200th year celebration. The project was later exhibited at The Wolverhampton Art Gallery and demonstrated at the Gadget Show Live (8-11 April 2010) at NEC Birmingham, UK. 
The Shift-Life installation was conceived when our lead artist Dew Harrison was invited by the Shrewsbury Museums Services, and funded by Arts Council England, to create a work related to Charles Darwin for the bicentenary of his birth in his birthplace of Shrewsbury. She was one of only 10 artists commissioned to make a work in response to Darwin's "On the Origin of Species" for the festival. For this she envisaged a real-world installation that would allow immediate access to a Darwinian virtual world of artificial creatures which users could affect via physical inputs. As a digital media artist with an interest in new technologies, her main focus is on the parallels between the human thinking process and computer processing where both are non-linear systems using semantic association to link items into ideas, and ideas into holistic concepts. She is also curious about perceptions and mind states as we move our attention between the created virtual world of the computer and the real-world we inhabit. Most of her work is interactive and considers viewer engagement as a participatory, and not as a passive activity.

Harrison uses digital thinking and technologies in her practice, which materializes as installations, interactive pieces, looped videos and online performances. Her work is concerned with the similarities of memory, mind and associative thinking held between computers and humans, where she constructs hypermedia systems of concept-based art by linking art's ideas into concepts. Earlier pieces focused on the complex and enigmatic art works and ideas of Marcel Duchamp, the forefather of Conceptual Art, who was immersed in the rapidly developing scientific understandings and new technologies of his time (1887-1968). Duchamp is singlehandedly responsible for the shift in art thinking from the aesthetics of the art object to the ideas held within that object, and for the understanding that the art object needs a viewer to "complete" it, through their mental engagement and interpretation. Such thinking led Harrison from the Modern to the Postmodern and, together with advances in computer technology, towards "interactive" art. Harrison began by creating interactive art pieces and now, in line with current art theory, builds "participatory" works in collaboration with programmers and designers. For "Shift-Life" she extended her practice of transposing Duchampian ideas into interactive hypermedia systems by transposing the "big idea" of Charles Darwin into a participatory installation. This Mixed Reality piece was to facilitate a holistic grasp of the necessary adaptation of life-forms in their struggle for survival in a volatile environment. It required the viewers to be physically engaged in activating the virtual ecosystem and then working with each other to keep it in balance.

From the Arts perspective, the project is an attempt to elucidate Darwin's thinking using holistic interaction interfaces. The bug-like creatures in the Mixed Reality installation are reminiscent of childhood and take the form of jelly sweets and allsorts. The creatures use a cartoony aesthetic to make manifest their artificiality; they are clearly hand drawn characters and not attempting to present an indexical trace of reality. Their visual roots in confectionary make clear the playful nature of the work and encourage interaction. The green/yellow jelly bugs are the plant-eating herbivores and the larger pink crab-like creatures are the carnivores. Darwin was born and 
spent his childhood in Shrewsbury, where he began his observation of natural life forms and started his vast collection of beetles. This boyish interest would last all his life and led to his great insights and contribution to science later on as an adult. In response to Darwin's idea, the aim of this "participatory" artwork was to create an "alternate" biological life as a set of artificial or virtual organisms that possess similar biological processes to their "real" counterparts - growth, reproduction, competition, and adaptation. The virtual life exists in a trophic relationship of predator and prey and includes sessile (rooted) and vagile (free moving) organisms. Animal intelligence was programmed into the virtual organisms to allow them survival strategies. The experiment involves the construction of an enhanced Mixed Reality-based environment which is connected with wireless sensors with environmental manipulators for altering the "climate" of the ecosystem. By bringing virtual "living" creatures into the physical world where they would seemingly respond to audience activity, we hoped to create a liminal space blurring the perceived virtual and real states to the point where our viewers might suspend the belief that these life forms were artificial, and thus engage with the work on a deeper level. Complementary details of the meanings behind the installation as an Art piece is available (Harrison, Ch'ng, Mount, \& Moore, 2009).

Here, a Mixed Reality interface is essential in our artwork as it encourages intuitive interactivity for visitors while the virtual world responds in real-time. Participants can physically manipulate a set of tools as everyday objects such as watering cans to radically alter the living conditions of the virtual creatures in their mixed virtual-real habitat. The virtual agents and their habitat are projected onto the installation space to create a sense of reality in the physical space, as if the creatures actually exist in the physical world. Participants alter the living conditions of the environment by pouring liquids to adjust the $\mathrm{pH}$ level and humidity of the soil, varying light conditions, and hammering to create earthquakes in the box, altering the conditions and reaction of the real-time agent simulation with immediate effect. The environmental change could be detrimental to the survivability of a certain species, or promote their growth and reproduction. These responses are dependent on the adaptation of their genotype, which describes their survival strategies and trophic networks.

Observation of the instant effect which a participant's actions had on the creatures and their ecosystem, projected onto the installation space proffered an understanding of how changes in environmental conditions induces reactive response as a result of their behaviors. Participants have direct control on tipping the balance between survivability and total destruction of the virtual-real ecosystem. Figure 1 is a relationship diagram of the Mixed Reality system described here, which illustrates how our artwork connects the relationships between participants, the interface sensors and the environment through its collection of artificial creatures. Participants in the artwork interact with physical sensors via physical props, sensors activate and send physical (electrical) and virtual (bits) signals to the virtual environment, virtual environment changes causing virtual agents to react, pixel information changes (virtual) and are fed back to the physical display. From a purely technical perspective, 
there has been a lack on the developmental aspects of integrating interactive artificial life and simulation of ecosystems with input that affects the simulation in real-time from participants via sensors, our system and the details of its implementation presented here acts as a formalization of an Mixed Reality system for the interactive art community, as well as the mainstream research community.

\section{FIGURE 1 HERE}

In the next section, we cover the brief background related to the present research. Section 3 documents the exhibit and participant reaction. Section 4 is the formalization of our Mixed Reality work, which covers the details of the implementation with behavioral mathematics for the artificial life and how they interface with the real world via environment sensors. Finally, we discuss our artwork, the interdisciplinary experience acquired during the creation the Shift-Life artwork, and what we have learned throughout the project.

\section{Mixed Reality, Art and Artificial Life}

The term "Mixed Reality" was first conceived in the seminal paper by Paul Milgram, "A Taxonomy of Mixed Reality Visual Displays" (Milgram \& Kishino, 1994), yet the two decades after the definition of Mixed Reality was set - what it includes, and its boundaries are still somewhat vague. We felt that the vagueness may be beneficial as it allows a broad range of interpretation, creativity and expression for the community. A particular interest of ours is how Art concerns can involve the use of Mixed Reality interfaces (Grasset, Woods, \& Billinghurst, 2007; Gwilt, 2009; Marshall, 2009), and the focus and content which drives our art installation is artificial life (Langton, 1997) which implies a simulation of ecosystems. The simulation of living creatures is of late a very popular area in virtual environments, yet Mixed Reality systems where simulation of artificial life and ecosystems affected by participants via sensors are still relatively unexplored in both art and other disciplines. The work here therefore, aims to formalize and outline the mechanisms involved in this particular strand of work.

Mixed Reality generally refers to a suite of technology that "overlays our real-world environment with digital, computer generated object" (Costanza, Kunz, \& Fjeld, 2009) covering diverse technological subjects from signal processing, computer vision, computer graphics, user interfaces, human factors, wearable computing, mobile computing, information visualization, and the design of displays and sensors (Costanza et al., 2009). Mixed Reality is differentiated with VR from the fact that in VR, participants are disembodied and completely immersed in an interactive virtual environment involving at least the sense of sight and contributing to the phenomenon known as presence (Lombard \& Ditton, 1997; Luciani, Urma, Marlière, \& Chevrier, 2004). Costanza et al. (Costanza et al., 2009) states that in Mixed Reality, the virtual aspects are equally dominant as the physical reality, this is again, in contrast with Augmented Reality (R. Azuma et al., 2001; R. T. Azuma, 1997) where virtual elements are less dominant in the display. The essence of Mixed Reality is the fusion of the real and the virtual, and this degree of fusion varies across projects. 
Mixed Reality is a field with many applications including that of the arts world. Artists create works with various media. Artistic "technology" has progressed through art specific media such as paint and canvas to those designed for other means and so artists now utilize digital technology in an exploratory manner. Some have extended the digital medium into the real world via combinations of physical and digital media. This combination merges the real with the virtual and can be categorized as a form of Mixed Reality media through which artistic ideas can be communicated. Artworks using Mixed Reality are not new. Grasset et al. (Grasset et al., 2007) provided a survey of artworks in the context of Mixed Reality, exploring artistic innovations via Mixed Reality technology. A deeper study which focuses on Mixed Reality and art practice studies the creation of illusion in computer aided performance (Marshall, 2009). Mixed Reality and artworks using mobile devices have also been studied (Gwilt, 2009). The context of meaningful encounters with artists and Mixed Reality is crucial and involves the look and feel, multimodal experience and interaction, and immersion as a subjective emotion, and game play scenarios (Misker \& van der Ster, 2010). Shaw et al.'s (Shaw, Kenderdine, \& Coover, n.d.) Web of Life and ConFIGURING the Cave allow participant's physical actions to be transposed, altering the aesthetics of the virtual imagery. Ulrike Gabriel's Breath (1992-1993) mentioned in (Barker, 2009), uses a combination of physical and digital information and processes to generate a particular aesthetic, co-mingling the physical and digital to generate aesthetics. While the many case studies of artists using Mixed Reality involve real humans but static, non-living computer generated 2D or 3D objects, we believe that the innovative inclusion of "living" virtual agents and concepts from AI will greatly enhance the experience of participants by exploiting more fully this digital encounter.

Artificial Life (Langton, 1997), the core driver of our artwork has been in widespread use in the entertainment industry since its potentials were demonstrated by Reynolds (Reynolds, 1987) in the synthesis of emergence in self-organizing systems such as bird flocks. Karl Sims’ Evolving Virtual Creatures (Sims, 1994) is another example. Artificial Life and Art have been discussed at length (Whitelaw, 2004). However, our survey reveals that this interest may not have been appropriately developed within the rather sparse Mixed Reality community. This is reflected in the lack of literatures formalizing its use. A seminal paper (Maes, 1995) attempted to contextualize the importance of believable and entertaining characters in entertainment with artificial life. Most artificial life characters mentioned both in the text and in literatures exist purely in the virtual environment (e.g., Prophet's work discussed at the end of the article) - at the extreme right-end of the virtual continuum.

In Artificial Life the simulation of an environment for supporting life is essential. Simulating life implies the understanding of the relationship between organisms and their environments, such as in Ecological Modeling (Gillman \& Hails, 1997). A particular branch of Ecological Modeling that abstracts away from state variable models is Individual Based Ecology (IBE), or generally referred to as Individual Based Model (IBM). 
Breckling et al. (Breckling, Müller, Reuter, Hölker, \& Fränzle, 2005) stated that "IBMs contrasts with common ecological models which frequently operate on the population level and represent a population as an overall state, thereby specifying rules how the overall state changes." Individual Based Model aims to develop theories of the adaptive behavior within the content of their population and environment and seeks to understand the mutual relationship between the adaptive behavior of individual and system-level properties of populations, communities, and ecosystems (Grimm \& Railsback, 2013). Individual Based Models are decentralized and is a bottom-up approach (Resnick, 1994) that is particularly suitable for our artwork.

Our project employs concepts from artificial life and ecology as drivers of "life" in our artwork for the Shift-Time festival, experimenting with Mixed Reality and applying the idea as an interactive simulation where voltage and silicon-based life-forms are brought into the physical environment and real-world environmental factors are merged with the virtual via hardware sensors that are partly controlled by participants.

\section{The Shift-Life Mixed Reality Art Exhibit}

To meet the commissioned development, and, in order to elicit an entertaining but partial understanding of the Darwinian view of life through our art interactive art, a collection of agent representations, together with an intuitive interface was required, mediated by tangible physical activity that participants could interact with. Here, we explain our approach in the making of Mixed Reality art.

\subsection{Agent Representations}

Our artist specifically chose the animator Sam Moore's work as she did not want the "creatures" to resemble real-life animals or microbes in any way, instead, preferring them to be flat graphics and non-scientific in their look. The agents also needed to be simple, fun, and to reference Darwin's childhood in Shrewsbury where he first began his interest in the natural world putting small beetles in boxes for observation. Sam Moore's animation style ticked all the boxes.

As such, the agent representations were developed as "bugs-in-a-box" "sweet" creatures with a view to making them as approachable as possible to a diverse audience (Figure 2). The images moved away from a computer-games visual aesthetic of hyper-reality and towards a deliberately non-digital, non-microbe, graphic and comic aesthetic. This positioned the animator's work in the realms of the overtly rather than covertly "made" and referenced a clear fantasy world instead of attempting photo-realism. The creatures were based on pick and mix sweets; the carnivore was a liquorice all-sort, the herbivore was a jelly sweet and the foliage (for shade, sustenance etc.) was based around a selection of penny sweets. The creatures were limited to two dimensions as they were to be observed from above. The completed representations of the agents and environments can be seen in the figure 4. 


\section{FIGURE 2 HERE}

\subsection{The Mixed Reality Art Installation}

The art installation (Figure 3) was made to a size that can accommodate small groups of people, families and individuals, accessible to both children and adult. The installation ran across two days at the Shrewsbury event and our observations and interviews showed that all our visitors, young and old actively contributed as participators by physically moving the box objects to change the parameters that affected the projected virtual world. Participants often remained in a state of reflection by passively observing others' actions, watching as "life" takes place in the virtual world. The algorithms implemented were self-sustaining and stable, without the need to intervene, as such it was visually mesmerizing as noted by our participants. There was room for contemplation where the virtual world could be understood as an analogy for human activity and its effect on global climate change within our own world. This became evident through our conversations with participants during the session. The tangible hands-on interface proved to be instinctive, attractive and informative on many levels, delivering a good example of collaboration between the Arts and Science.

As our art is "alive", there were permutations of scenarios documented from the exhibition demonstrating the many facets of events that could possibly happen, these were some of them:

- As they poured water, for instance, the humidity would alter and some plants may die back, this would mean less food for the herbivore green jelly sweet bugs, and consequently less bugs to eat for the pink carnivores.

- Switching the lamp on would dry out the atmosphere and enable plants to grow again, however too much "sun" might be detrimental to the point of wiping out the carnivores entirely! They could, in fact, become extinct due to their reproduction method of cloning, unlike the egg laying herbivores. When this occurred, we had to re-start the program to reassure the smaller children that they weren't responsible for a complete genocide.

- Pouring vinegar from a watering can would "feed" the poisonous plants, toxic to all the creatures, but this could be remedied by pouring baking soda liquid and restoring the plant balance, the herbivores' food.

- Hammering on the box sent the carnivores into panic mode and they would run for cover under the trees. 


\subsection{Participant Reactions}

Once the piece was completed and on show, the viewers' slippage between virtual and real states became apparent, even though the set-up was basic with plastic watering cans etc., and the creatures were comic brightly colored line drawings, the viewers' talked about them as though they were alive. Their conversations illustrate their understanding of how the animals had to "adapt to survive" the climate changes that were being introduced into their ecosystem. But more interestingly, we also saw how this piece worked on a higher level as a signifier for human activity - climate change and the earth:

"What happens if you change the light, do the creatures disappear?" someone else requested that the lamp be brought nearer the box, "oh, they're all dying!"

"So, if the Earth gets too close to the sun, we'll all die?" "You've completely changed their planet, they're all dying, it's not in balance." This meant that they were now extinct "like the dinosaurs?" The participants asked us to re-start the system to bring the creatures back into a sustainable environment, which we did. "This could be the Earth that you've wiped out, we can't re-boot the earth!'

The participants expressed similar sentiments, "the simulation was self-sustaining, stable and all found the installation visually mesmerizing". As creators of the system, we ourselves were occasionally surprised by the emergent behavior witnessed during the exhibition - simple rules can indeed produce complex behaviors, for "the whole is greater than the sum of its parts" $\sim$ Aristotle.

\section{FIGURE 3 HERE}

Figure 3 shows various scenarios during Shift-Time Shift-Life art exhibition. In A and B, young participants can be seen adjusting the "sunlight" and adding moisture to the virtual environment to help with the plant growth. In C, participants added vinegar into the watering in order to adjust the $p H$ level of the virtual soil for helping with the growth of certain species of plants. In D, a participant is seen pointing to a dying creature. E shows a participant hammering the sides of the sandpit box in order to create a virtual earthquake. Finally in F, crowds are seen gathering around the sandpit, mesmerized by the dynamics of the flourishing ecosystem when the environment is appropriately moderated through the Mixed Reality props.

Figure 4 contains screenshots of the virtual environment when participants interacted with the Mixed Reality system. Each figure is at a different period of the simulation showing different scenarios. The figure in the top left shows carnivores emerge from the canopies after an earthquake (activated by participants hitting the "earthquake" sensor with a toy hammer). In the top right are newly hatched herbivores heading towards the clusters of edible plants. At the bottom left are carnivores in a feeding frenzy. Two herbivores in the same figure have just died (multi-shaded blobs) due to attacks by a carnivore. At bottom right are herbivores clustering around the group of plants. Some carnivores have died due to senescence and from ingesting a herbivore made toxic by ingesting poisonous plants. 


\section{FIGURE 4 HERE}

\section{Implementation of the Mixed Reality Interface}

Mixed Reality interfaces generally refer to systems which augment reality by overlaying real-world environments with computer-generated objects via headsets or digital displays. Our research experimented with an inverse of this concept by projecting computer generated agents and environment into the physical world and feeds environmental factors (temperature, sunlight, humidity, soil $p H$, and earthquake) back to the virtual environment. This allows a mixed-mutual relationship between the real and the virtual - virtual agents sense our environment while human participants interact with them. Our approach is particularly suited to relational artworks involving multiple participants in public spaces. The more complex task here is the development of an artificial life ecosystem (Section 4.3), the "life" of our artwork, which will be the body of technical details covered in this section. We believe that the formalization of our Mixed Reality approach will enable more creative approaches in the future when the ecology of "living" objects becomes necessary. In this context, our lead artist has since expanded her practice towards notions of creating "living" virtual Duchampian art objects endowed with animal behaviors, which might inter-relate to allow new insights into this body of work.

\subsection{The Shift-Life Interactive Art Installation}

The Mixed Reality system comprised of a $1.2 \mathrm{~m}^{2}$ box filled with polystyrene beads held under a muslin sheet and surrounded by a set of manipulative tools. A projector fixed to the ceiling of the installation projects real-time rendering of the virtual agents and its environment onto the muslin sheet. The interactive tools are made up of a foam-based hammer used for simulating earthquakes; two watering cans were used for altering the humidity and $\mathrm{pH}$ level of the soil, and a light source for altering the luminance to affect both the temperature and the light/shade of the environment. Figure 5 illustrates the specifications with a cross-section diagram.

\section{FIGURE 5 HERE}

\subsection{Environmental Sensors}

The sensor system consists of the following devices:

- An attached Oak USB 3-axis accelerometer hidden underneath a paper icon attached to the side of the bug box and a toy hammer with which to actuate the sensor. The output of the sensor was normalised and an exponential weighted moving average applied to smoothen the response from the transducer;

- An Oak USB luminosity sensor, actuated by an angle-poise lamp;

- An Oak USB relative humidity sensor, protected by a Gortex membrane, actuated by pouring water into the bug box, and 
- $\quad$ A Phidgets USB $p H$ sensor, actuated by pouring vinegar and bicarbonate solutions into the sandpit.

The sensor network was centralised and reported back to the system. Software objects in the environment receive the signals and adjusts the virtual environment accordingly, which affects the virtual agents in a feedback loop. A number of optimisations were made to enable the application to appear to be responding immediately, i.e., unnecessary sensor signals and logs were suppressed, and each datum only reported back to the ecosystem if it differed from the previous reading by a threshold.

\subsection{Agents and Environments}

This elaborate section details the implementation of the agent-based model and virtual environment, which drives the virtual creatures in our Mixed Reality art installation. The sub-sections describe in detail the behavioral mathematics and algorithms, which are foundational to the implementation of our Mixed Reality system. We believe that by outlining our approach in detail, our system can be replicated, and used by researchers wishing to extend our work in other application areas. For the purpose of standardizing the presentation of the agent-based model for our readership, the format of the technical presentation of the model in this section follows that of the Individual-Based Modeling (IBMs) technique in the Ecological Modeling community (Grimm et al., 2006).

\subsubsection{Purpose}

The purpose of the agent-based model used in our system is to generalize, and to create a collection of extensible living entities which can be interacted with, in real-time, via physical inputs.

\subsubsection{State variables and scale}

The model we used for our virtual creatures is a predator-prey ecosystem simulation and comprises a hierarchical level of individual, meta-population, community and environment. There are three types of agents. The first two are vagile - herbivore, carnivore, and the third is sessile - a vegetation type, these are edible herbs, canopies, and poisonous plants. The agents are short-lived (60 seconds minimum and 150 seconds maximum), this is essential for public exhibition where a large population of visitors are expected. The short life-span of the creatures allow our viewers to see a fully scenario of the simulation in a few minutes of viewing, but people generally stayed for half and hour or more. The general behavior of each organism is its survivability and the reproduction of progenies. The survival of the entire ecosystem depends on the balance of the organisms that inhabit the landscape. If the predator out-grows the prey, an imbalance occurs and the system perishes. If the prey out-grows the edible plants, the food is scarce and the system is at a dilemma. If the canopies (large trees) over-reproduce, the predator has little space left to hunt. If the poisonous plants outgrow the vegetation, more and more herbivores are toxic; this toxic reaction kills the carnivores when they eat the herbivores. When the environment is suitable, the plant species that found its niche reproduces better (poison plants love high $\mathrm{pH}$ 
levels). The difficulty of such an artificial life development is the maintenance of equilibrium. The "fun" part of the exhibit is when users interact with the system by increasing any of the environmental factors via our sensor network: temperature, sunlight, humidity, $p H$ level.

Herbivore and carnivore individuals are characterized by:

1. Dynamics - speed (hunting, fleeing), eyesight, field-of-view (FOV)

2. Physique - age, deterioration (affects the maximum age), energy, hunger threshold, flesh index

3. Behavior - impulse, safe distance (security boundary), feeding distance

4. Reproduction - number of progenies, and sexual maturity

5. Ecology - adaptability to sunlight, temperature, seismic vibrations, and humidity.

Vegetation is characterized by these traits: their energy, resource index, seed count, reproduction age, dispersal distance, and ecology - adaptability to sunlight, temperature, soil $p H$, availability of space and humidity.

The population is characterized by size (the number of individuals in a given species) and the community interacts within their trophic network. Populations can be controlled (culled) when a threshold is reached. The highest hierarchical level in the model is the environment, which is discrete, i.e., the smallest unit of movement is a single pixel. Abiotic factors depend on user-interaction and include temperature, sunlight, $p H$ level, humidity, and seismic vibrations (earthquakes). Environmental factors can both be controlled by both on-screen sliders (widgets) and hardware sensors. Table 1 shows an overview of the processes, parameters and individual genotype of the model. The symbols used represent variables in the equations in the Submodels section (4.3.7). It is important to note that these variables are based on the principal author's literature review, and observation of animal behavior in the wild and in captivity, which were translated into a relative measure during our development. Whilst the variables were meant to reflect only the behaviors of our imaginary creatures, they do have semblance with real world organisms.

\section{Table 1 - Overview of processes, parameters, and individual genotypes of the model}

\section{Global Parameters}

Aging of agents (per seconds)

Symbols \& Value(s)

Simulation time steps (dynamics)

$1000 \mathrm{~ms}$

Rendering Frames Per Seconds (FPS)

$\Delta t=0.012 \mathrm{~s}$

12

Abiotic Parameters (depends on where the sensors are placed: indoor/outdoor)

\section{Temperature}

Sunlight

$\mathrm{pH}$

Humidity

Seismic vibrations
Symbols \& Value(s)

$25^{\circ} \mathrm{C}$

0.6

8

0.5

0 or 1

Symbols \& Value(s)

Carnivore Parameters

Age at start of simulation (seconds)

Maximum age

Deterioration Time (a threshold reached where the creature deteriorates)

Speed (pixels)

$A_{j}=60$

10

$\sigma=2$

$\varepsilon=2$

$\varepsilon=3$

Thrust

Hunting Thrust 


\section{Fleeing Thrust}

Thrust Limit

Friction

Rotation Angle

Eyesight

Field of View

Energy at start of simulation

Energy Loss (when moving)

Energy Rest Threshold (at a point when agent needs rest)

Energy used in predation

Hunger threshold (depletion of energy results in hunger)

Flesh index

Impulse range (pixels)

Impulse

Safe distance (from predation)

Feeding distance (prey captured at this distance)

Progenies

Next reproduction age (reproduced only after $n$ seconds)

Mature Age Ratio (reproduced when $>=$ age*MatureAgeRatio)

Sunlight ( $\mathrm{L}=$ lower range, $\mathrm{P}=$ preferred, $\mathrm{U}=$ upper range)

Temperature $\left({ }^{\circ} \mathrm{C}\right)(\mathrm{L}=$ lower range, $\mathrm{P}=$ preferred, $\mathrm{U}=$ upper range $)$

Humidity ( $\mathrm{L}=$ lower range, $\mathrm{P}=$ preferred, $\mathrm{U}=$ upper range)

Seismic vibration (Earthquake)

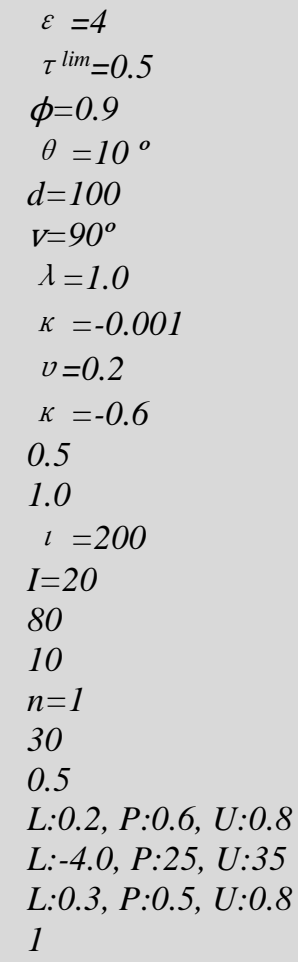

Symbols \& Value(s)

\section{Herbivore Parameters}

\section{1}

Initial size of creature at birth

Rate of growth

Maximum age

Deterioration Time (a threshold reached where the creature deteriorates)

Speed (pixels)

Thrust

Hunting Thrust

Fleeing Thrust

Thrust Limit

Friction

Rotation Angle

Eyesight

Field of View

Energy at start of simulation

Energy Loss (when moving)

Energy Rest Threshold (at a point when agent needs rest)

Energy used in predation

Hunger threshold (depletion of energy results in hunger)

Flesh index

Impulse range (pixels)

Impulse

Safe distance (from predation)

Feeding distance (prey captured at this distance)

Progenies

Next reproduction age (reproduced only after $n$ seconds)

Mature Age Ratio (reproduced when $>=$ age*MatureAgeRatio)

$\varsigma^{0}=0.5$

$g=5.0$

$A=100$

10

$\sigma=2$

$\varepsilon=3$

$\varepsilon=3$

$\varepsilon=8$

$\tau^{\lim }=0.5$

$\phi=0.9$

$\theta=10^{\circ}$

$d=250$

$v=90^{\circ}$

$\lambda=1.0$

$\kappa=0.0005$

$v=0.0$

$\kappa=0.5$

0.8

$K=1.0$

$l=200$

$I=5$

50

3

$n=5$

15

0.3

L:0.1, P:0.6, U:0.8

$L:-4.0, P: 25, U: 35$

$L: 0.3, P: 0.5, U: 0.8$

1

Symbols \& Value(s)

\section{Vegetation Parameters}

1

Maximum age 1

A $=70$

15

Energy

$\rho=1.0$

$K=1.0$

$s=5$ 
Reproduction age

Dispersal distance (pixels)

Sunlight ( $\mathrm{L}=$ lower range, $\mathrm{P}=$ preferred, $\mathrm{U}=$ upper range)

$D=130$

Temperature $\left({ }^{\circ} \mathrm{C}\right)(\mathrm{L}=$ lower range, $\mathrm{P}=$ preferred, $\mathrm{U}=$ upper range)

Soil $(\mathrm{P}=$ preferred, $\mathrm{U}=$ upper range)

$\mathrm{pH}$ ( $\mathrm{L}=$ lower range, $\mathrm{P}=$ preferred, $\mathrm{U}=$ upper range)

Space $(\mathrm{P}=$ preferred, $\mathrm{U}=$ upper range)

Humidity ( $\mathrm{L}=$ lower range, $\mathrm{P}=$ preferred, $\mathrm{U}=$ upper range)

$L: 0.1, P: 0.5, U: 0.8$

$L: 18.0, P: 26, U: 38$

$P: 0.5, U: 0.8$

L:5.0, P:8.0, U:10.0

$P: 0.6, U: 1.0$

$L: 0.3, P: 0.5, U: 0.7$

\section{Canopy Parameters}

Symbols \& Value(s)

Age at start of simulation (seconds)

\section{1}

Maximum age

$A=150$

Culling (maximum population)

10

Energy

$\rho=1.0$

Resource index

$K=1.0$

Seed count

$s=5$

Reproduction age

25

Dispersal distance (pixels)

$D=250$

Sunlight ( $\mathrm{L}=$ lower range, $\mathrm{P}=$ preferred, $\mathrm{U}=$ upper range)

Temperature $\left({ }^{\circ} \mathrm{C}\right)(\mathrm{L}=$ lower range, $\mathrm{P}=$ preferred, $\mathrm{U}=$ upper range)

$L: 0.1, P: 0.8, U: 1.0$

Soil $(\mathrm{P}=$ preferred, $\mathrm{U}=$ upper range)

$\mathrm{pH}$ ( $\mathrm{L}=$ lower range, $\mathrm{P}=$ preferred, $\mathrm{U}=$ upper range)

Space $(\mathrm{P}=$ preferred, $\mathrm{U}=$ upper range)

Humidity ( $\mathrm{L}=$ lower range, $\mathrm{P}=$ preferred, $\mathrm{U}=$ upper range)

$L: 15.0, P: 30, U: 45$

$P: 0.4, U: 0.6$

L:1.0, P:7.0, U:10.0

$P: 0.28, U: 0.5$

$L: 0.3, P: 0.5, U: 0.7$

\section{Poison Plant Parameters}

Symbols \& Value(s)

Age at start of simulation (seconds)

1

Maximum age

A $=50$

Culling (maximum population)

15

Energy

$\rho=1.0$

Resource index

$K=1.0$

Seed count

$s=3$

Reproduction age

10

Dispersal distance (pixels)

Sunlight ( $\mathrm{L}=$ lower range, $\mathrm{P}=$ preferred, $\mathrm{U}=$ upper range)

$D=160$

Temperature $\left({ }^{\circ} \mathrm{C}\right)(\mathrm{L}=$ lower range, $\mathrm{P}=$ preferred, $\mathrm{U}=$ upper range)

$L: 0.1, P: 0.4, U: 0.7$

Soil $(\mathrm{P}=$ preferred, $\mathrm{U}=$ upper range)

$L: 16.0, P: 27, U: 33$

$\mathrm{pH}$ ( $\mathrm{L}=$ lower range, $\mathrm{P}=$ preferred, $\mathrm{U}=$ upper range)

Space ( $\mathrm{P}=$ preferred, $\mathrm{U}=$ upper range)

Humidity ( $\mathrm{L}=$ lower range, $\mathrm{P}=$ preferred, $\mathrm{U}=$ upper range)

$P: 0.5, U: 0.8$

L:6.0, P:9.0, U:14.0

$P: 0.6, U: 1.0$

$L: 0.3, P: 0.5, U: 0.7$

\subsubsection{Process overview and scheduling}

Time is discrete steps in the simulation and the aging of the agents proceed every seconds. Within each second, certain processes (simple rules) occur - state changes, growth, interaction, adaptation, feeding/fleeing, reproduction and inheritance, and senescense. Figure 6 and 7 are Finite State Machines (FSM) of the Carnivore and Herbivore agents. The processes for each autonomous agent are specified in the pseudocode below.

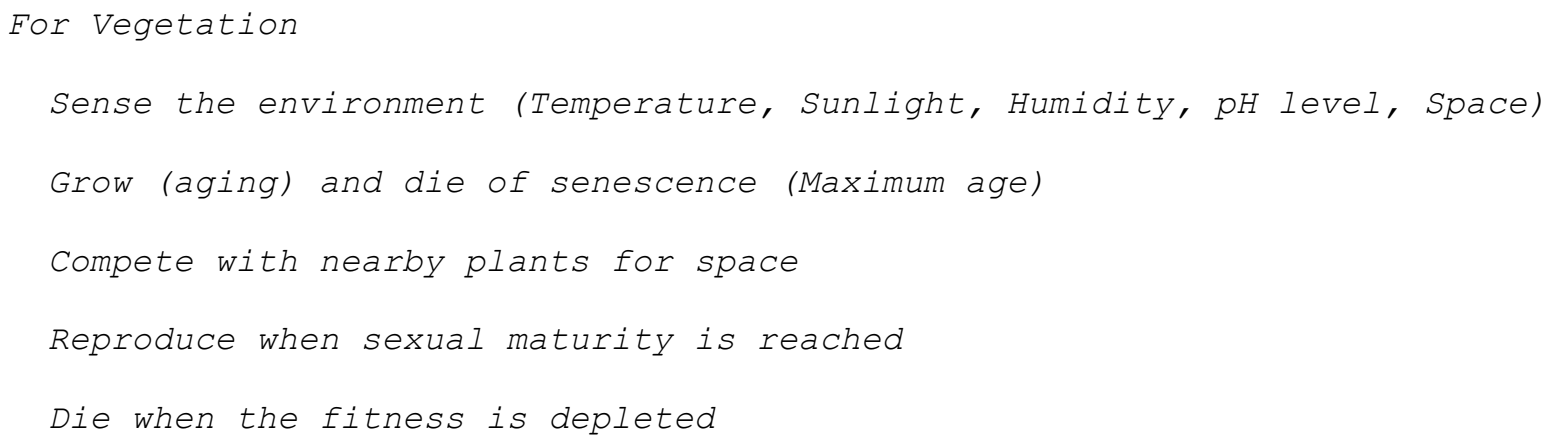




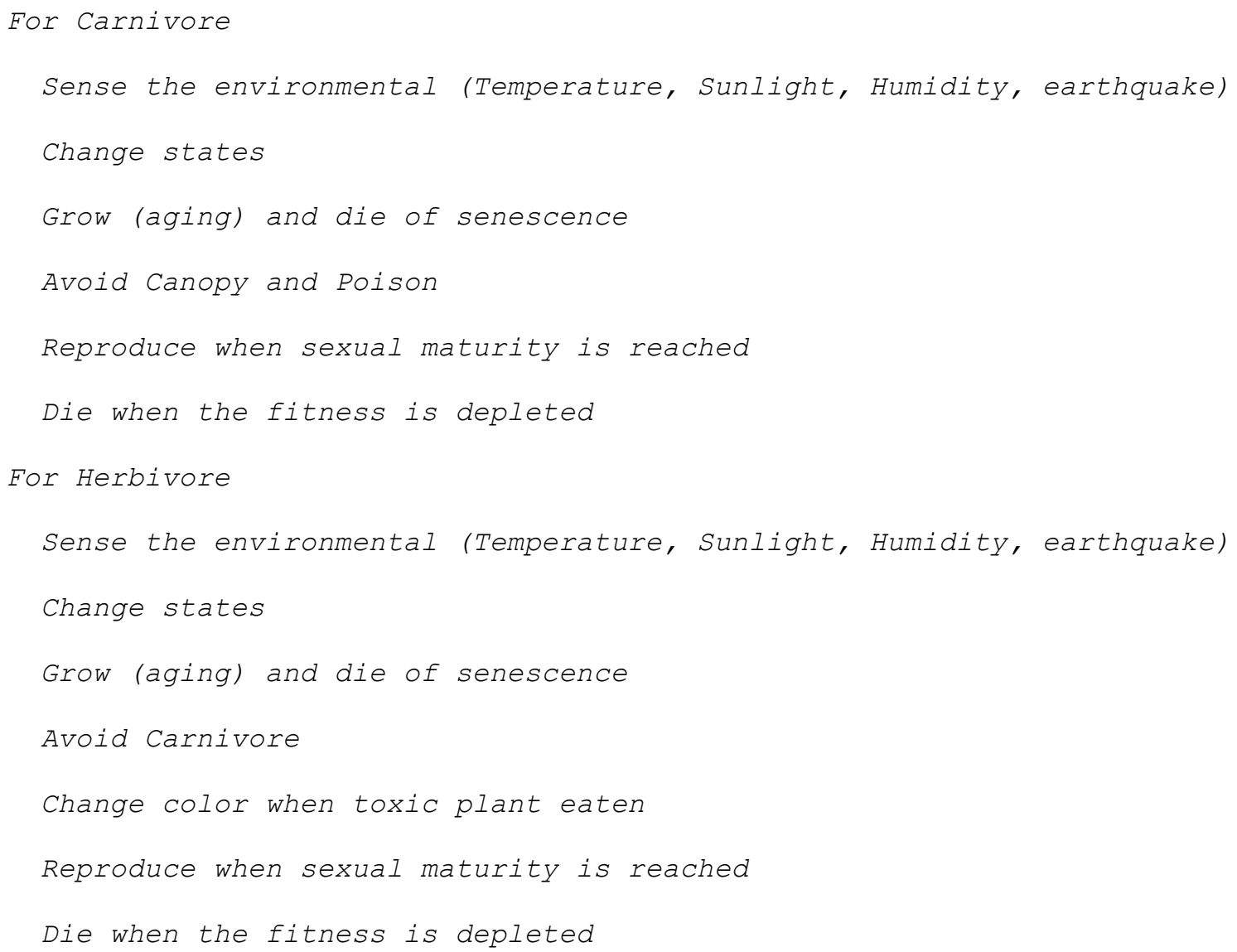

FIGURE 6 HERE

\section{FIGURE 7 HERE}

\subsubsection{Design concepts}

The concepts presented below are central to simulating an ecosystem of virtual creatures which interact amongst themselves and with the environment affected by physical inputs. Since our model is a complex system, explicitly presenting these concepts will also help with the observation of individual and collective behaviors during simulation time.

Emergence: Emergence does not occur at the population level as there are no interactions between the same species of agents. At the community level, certain emergent phenomena are observable due to the interactions between predator, prey, vegetation (food and protection), and abiotic factors.

Adaptation: Adaptation in the model refers not to the general adaptation in evolutionary time-scale but is tolerance to biotic and abiotic factors that have already been developed.

Fitness: The fitness of each organism is measured by the Adaptability Measure (Ch'ng, 2007). The creatures are affected by three abiotic factors: temperature, sunlight and humidity and the plants are affected by six factors: temperature, sunlight, soil, humidity, $p H$ level, and space. The temperature, sunlight, humidity and $p H$ level are streamed from electronic sensors installed at strategic locations in the virtual bug box. Soil conditions are unchanged and are based on height fields (certain regions are more habitable). The availability of 
space for the plants depends on the number of plants growing within that space. Fitness-seeking are not modeled. The fitness measure is provided in later sections and is a product of the Adaptability Measure.

Prediction: Estimation of future consequences of agent decisions is not modeled.

Sensing: Agents are aware of their own age-related mechanisms (reproduction, senescence), other agents that has direct impact on their survivability, and the abiotic factors.

Interaction: Individuals do not interact within their species. Carnivores interact with herbivores via predation. Carnivores avoid canopies, which are protection for herbivores. Herbivores interact with all three types of vegetation. Edible and poison plants provide energy but poison plants provide an additional chemical which, if ingested by carnivores causes death.

Stochasticity: Stochasticity is implemented in the distribution of seeds (angle and bounded distance), and the impulses of the movement thrusts of the vagile agents.

Collectives: Individuals are not grouped into collectives or social groups.

Observations: Scientific observation of the model itself is unnecessary as this is an interactive art simulation.

\subsubsection{Initialization}

At the start of simulation, all agents are randomly distributed in the landscape using the default parameters listed in Table 1. Similarly, abiotic factors are initialized and read from the hardware sensors, which depends on the current temperature at the place of installation. The environment varies depending on where the sensors are - outdoors, or within an enclosed space.

\subsubsection{Input}

Abiotic parameters are directly read from the hardware sensors. The raw data is polled and processed every $0.012 \mathrm{~ms}$. This corresponds to the simulation cycle of the agents, ensuring a continuous flow of abiotic information to the biotic components.

\subsubsection{Submodels}

This section explains in detail all submodels and parameterization representing the processes listed within Section 4.3. The section covers the mathematical structure of the vegetation behavior and the dynamics of the vagile agents.

\subsubsection{Vegetation Behavior}

Vegetation behavior is a simplified version of an agent-based model (Ch'ng, 2009). The simplified model has minor changes to suit the difference in sensing of abiotic factors by agents. 
Sensing the Environment

The vegetation agents sense the environment via receptors. The abiotic factors that the agents take into account are temperature, sunlight, humidity and soil $\mathrm{pH}$. Biotic factors include the competition for space and the risk of being consumed by Herbivores. Each abiotic factor is measured by the Adaptability Measure $(A M)$ (Ch'ng, 2007) and contributes to the fitness measure.

\section{Fitness Measure and Death}

The $A M$ measures four abiotic factors and a biotic factor to generate the fitness for individual agents $i$ at time $t$ (Equation 2) in each simulation cycle:

$$
f_{i}^{t}=\varphi_{i}(C T S \eta)_{i}^{t}
$$

Where the output of $A M$ for each fitness related to biotic or abiotic interactions are computed: $\varphi_{i}$ is the interaction fitness of the $p H$ level of the soil for plant $i, C_{i}^{t}$ is the only biotic local interaction fitness of the current condition $c_{i}^{t}$ (Equation 2) at time $t, T_{i}^{t}$ is the interaction fitness of the plant related to the temperature, $S_{i}^{t}$ is the fitness affected by the sunlight and $\eta_{i}^{t}$ is the fitness of the plant in the current humidity. The interaction of the factors is a logical way for deciding the fitness of the plant. The variable Resource Index $\rho$, defined as the storage of energy is decremented $\kappa$ unit (Table 1) in the condition in Equation 2. Death occurs when $\rho_{i}^{t+\Delta t} \leq 0.0$

$$
\rho_{i}^{t+\Delta t}=\rho_{i}^{t}-\left(\kappa_{i}\left[f_{i}^{t} \leq 0.0\right]\right)
$$

\section{Competition}

The collective occupation of the space used by the competing plants contributes to the accumulated space $c_{i}^{t}$ at time $t$ for the plant $i$ in Equation 3. Competition for space is defined as an interaction. A plant interacts with its neighbor in the condition,

$$
\begin{aligned}
& c_{i}^{t}=\sum_{i=1}^{n} P_{i}\left[\sqrt{\left(O_{x}^{t}-u_{x}^{t}\right)^{2}+\left(O_{y}^{t}-u_{y}^{t}\right)^{2}}-\left(O_{\text {size }}^{t}+u_{\text {size }}^{t}\right)<0\right]\left[O_{\text {size }}^{t} \geq u_{\text {size }}^{t}\right]\left[O_{\text {age }}^{t} \geq u_{\text {age }}^{t}\right], \\
& \text { for } 0 \leq c_{i}^{t} \leq 1
\end{aligned}
$$

Where $n$ is the number of competing plants, and $P_{i}$ is the effective space used by a single plant. $P_{i}=0.05$ if the undergrowth species (vegetable or poison) competes against it's own species, $P_{i}=0.12$ if the undergrowth compete against a canopy, and $P_{i}=0.2$ among canopy competition. The differences in $P_{i}$ adjusts the space so that the canopies are not too crowded together. $O_{x, y}^{t}$ is the position of the competitor and $u_{x, y}^{t}$ is 
the position of the source plant at time $t . O_{\text {size }}^{t}$ and $u_{\text {size }}^{t}, O_{\text {age }}^{t}$ and $u_{\text {age }}^{t}$ are respectively the diameter and the age of the two competing plants.

\section{Growth and Reproduction}

Growth and reproduction uses time as a measurement. Growth is by aging, plants age every seconds and senescense occurs when the maximum age is reached. Reproduction for Canopy and Vegetable depends on the parameter Reproduction Age and the number of seeds $s$. Poison plant reproduces quicker on the high $p H$ level of the soil. The pseudo code below describes the process, the parameters for the variables below is listed in Table 1:

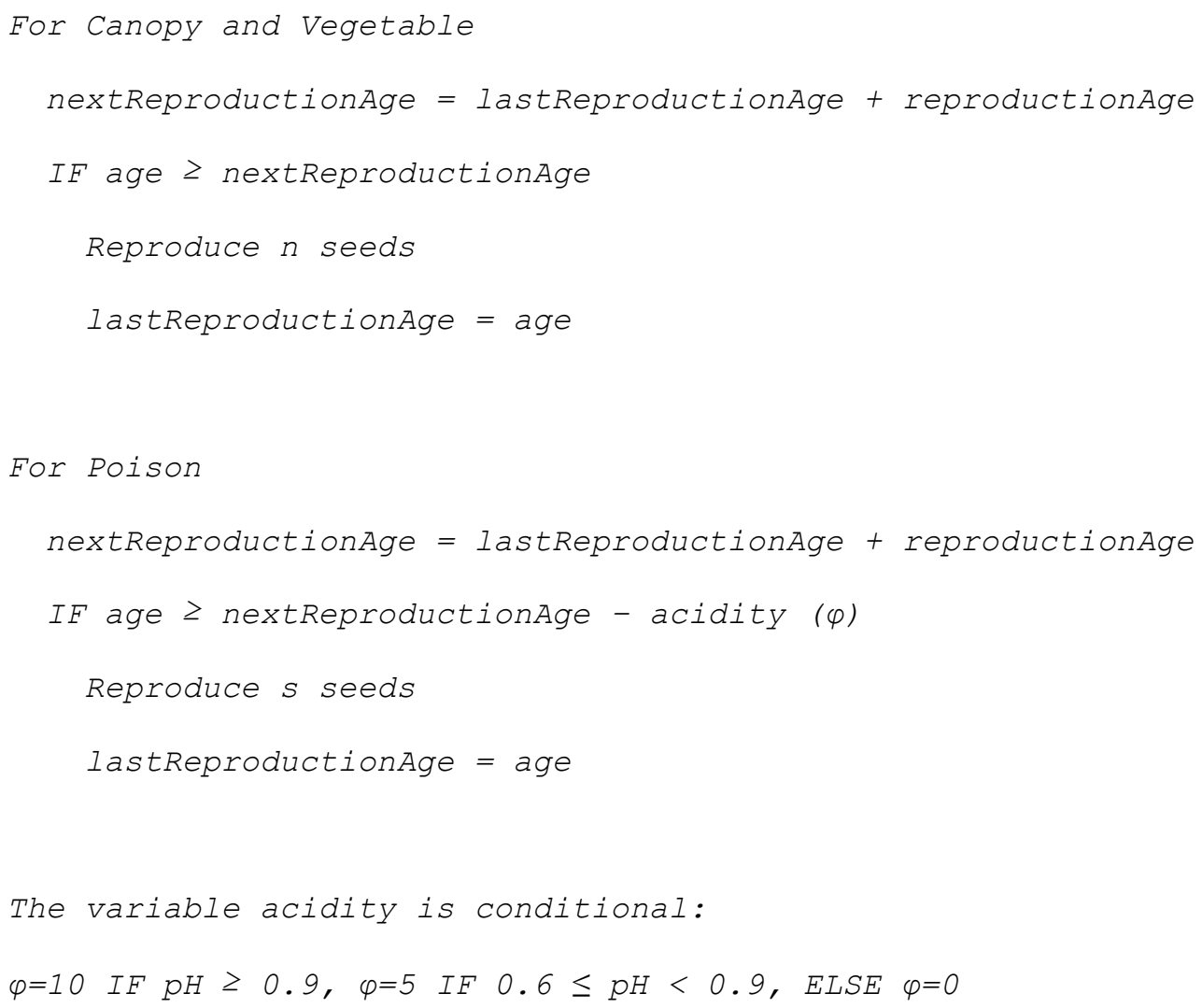

Reproduction distance and direction is stochastic:

$$
\begin{aligned}
& x_{s}=x_{i}+D_{i} X \cos \beta . \\
& y_{s}=y_{i}+D_{i} X \sin \beta .
\end{aligned}
$$

Where $x_{s}$ and $y_{s}$ are respectively the $\mathrm{x}$ and $\mathrm{y}$ position of the seed, $x_{i}$ and $y_{i}$ are respectively the $\mathrm{x}$ and $\mathrm{y}$ position of the parent plant, $X$ is a function from $\mathbf{R}$ to $[0,1]$ and is a stochastic variable. $D_{i}$ is the maximum dispersal distance of the parent plant (see Table 1), finally $\beta$ is a random angle in the interval $[0,360]$. 


\subsubsection{Carnivore and Herbivore Behavior}

\section{Sensing the Environment}

The vagile agents sense the environment via receptors. The abiotic factors that the agents take into account are temperature, sunlight, and humidity. Each abiotic factor is measured by the Adaptability Measure (AM) (Ch'ng, 2007) and contributes to the fitness measure.

\section{Fitness Measure and Death}

The AM measures each environmental factor, which is combined with other factors to generate the fitness $f_{j}^{v}$ for each individual agent $j$ (Equation 6) in each simulation cycle:

$$
f_{j}^{t}=(T S \eta)_{j}^{t}
$$

Where the output of $A M$ for each fitness related to abiotic interactions is computed: $T_{j}^{t}$ is the interaction fitness of agent $j$ at time $t$ in the current temperature, $S_{j}^{t}$ refers to the interaction fitness with sunlight and $\eta_{j}^{t}$ is the fitness in the current humidity. When $f_{j} \leq 0.0$ the agent dies.

\section{Growth and Reproduction}

Growth and reproduction uses time as a measurement. Growth is defined by aging - the agent ages every seconds and senescense occurs when the maximum age is reached. Graphically, the size of the Herbivore agents has the following ratio, Carnivore size does not change.

$$
\varsigma_{j}^{t+\Delta t}=\varsigma_{j}^{0}+\frac{1}{1+e^{g\left(1-A_{j}^{-1} 4 t\right)}}
$$

Where $g$ is a constant $(g=5.0)$ is the Rate of Growth to reach full size, it is the growth spurt, $A_{j}$ is the Maximum Age of the agent, and $\varsigma_{j}^{0}$ is the Initial Size of creature at birth.

Reproduction depends on the parameter Mature Age Ratio, Reproduction Age and the number of Progenies $n$. The progenies appear from below the agents when they are reproduced. The pseudo code below describes the process, the parameters for the variables below is listed in Table 1:

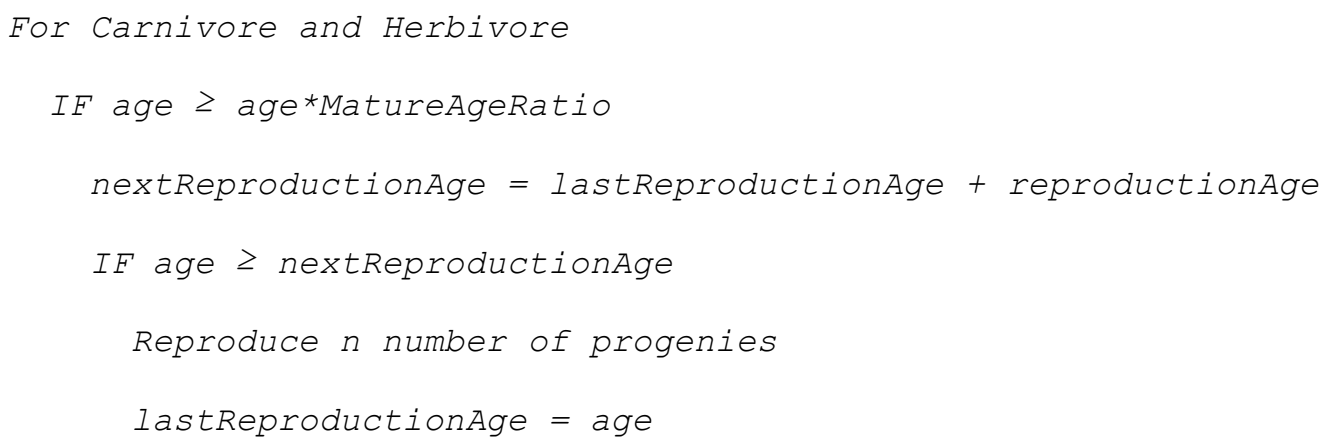




\subsubsection{Behaviors and Dynamics of Carnivores and Herbivores}

These behaviors are our approach in defining the movement dynamics and behaviors of our virtual creatures, such as chase and evade, roaming and scavenging, and energy gain and loss. These are foundational formulae, essential to the implementation of our simulation, which bring the virtual creatures to life. The formulae can be extended for application in other research.

\section{Movement When Targeting Another Agent}

During one simulation time step, the steering behavior of the agent position updates with the speed, thrust (to propel the body forward) and heading,

$$
\begin{aligned}
& x_{j}^{t+\Delta t}=x_{j}^{t}+\sigma_{j} \tau_{j}^{t} \cos \left(\omega_{j}^{t}\right) . \\
& y_{j}^{t+\Delta t}=y_{j}^{t}+\sigma_{j} \tau_{j}^{t} \sin \left(\omega_{j}^{t}\right) .
\end{aligned}
$$

Where $\left[\begin{array}{cc}x_{j}^{t+\Delta t} & y_{j}^{t+\Delta t}\end{array}\right]$ is the vector of agent $j$ with speed $\sigma_{j}$ (length of the vector) and Thrust $\tau_{j}^{t}$ (changes only during the roaming state, see Equation 20) at time $t+\Delta t$. The heading $\omega_{j}^{t}$ (orientation of the vector) depends on the position of the target agent $j$,

$$
\omega_{j}^{t}= \begin{cases}+\theta_{j} & \text { if } u_{j k}>1 \\ -\theta_{j} & \text { if } u_{j k}<-1 .\end{cases}
$$

Where $\theta_{j}$ is the agent heading. Avoidance behavior is defined below by simply swapping the sign associated with the heading,

$$
\omega_{j}^{t}= \begin{cases}-\theta_{j} & \text { if } u_{j k}>1 \\ +\theta_{j} & \text { if } u_{j k}<-1\end{cases}
$$

$u_{j k}^{t}$ and $v_{j k}^{t}$ are the visibility (Eyesight) from agent $j$ to target agent $k$ with the distance between the agents in the $x$ and $y$ component $x_{j k}^{t}$ and $y_{j k}^{t}$ at time $t$,

$$
\begin{aligned}
& u_{j k}^{t}=x_{j k}^{t} \cos \left(-\omega_{j}^{t}\right)+y_{j k}^{t} \sin \left(-\omega_{j}^{t}\right) . \\
& v_{j k}^{t}=-x_{j k}^{t} \sin \left(-\omega_{j}^{t}\right)+y_{j k}^{t} \cos \left(-\omega_{j}^{t}\right) .
\end{aligned}
$$

Where,

$$
\begin{gathered}
x_{j k}^{t}=x_{k}^{t}-x_{j}^{t} . \\
y_{j k}^{t}=y_{k}^{t}-y_{j}^{t} .
\end{gathered}
$$

The visibility of the agent from agent $j$ to $k$ and their position $x$ and $y$ at time $t$, 


$$
\left\|d_{j k}^{t}\right\|=\sqrt{\left(x_{j}^{t}-x_{k}^{t}\right)^{2}+\left(y_{j}^{t}-y_{k}^{t}\right)^{2}} \text {. }
$$

The target agents are visible to the hunting agents at $90^{\circ}$ field of view,

$$
\vartheta_{j}=\left[v_{j k}>0\right]
$$

Where $\vartheta_{j}$ is the decision to hunt (move to) the target.

\section{Movement during the ROAM State}

When in the roaming state, both Carnivore and Herbivore uses their impulses to navigate. The impulse to turn either right or left and the thrust to move forward in short bursts depends on Equations 18 and 19,

$$
\begin{gathered}
\omega_{j}^{t}=\left\{\begin{array}{ll}
+\theta_{j} & \text { if } X \iota_{j}<\mathrm{I}_{j} \\
-\theta_{j} & \text { if } X \iota_{j}>\mathrm{I}_{j}
\end{array} .\right. \\
\tau_{j}= \begin{cases}\varepsilon_{j} & \text { if } X \iota_{j}<\mathrm{I}_{j} \\
0 & \text { otherwise }\end{cases}
\end{gathered}
$$

Where $\omega_{j}$ is the heading of the agent $j$ with steering angle $\theta_{j}$ at time $t$, the thrust $\tau_{j}$ as the decision to propel the body forward with force $\varepsilon_{j}, X$ is a function from $\mathbf{R}$ to $[0,1]$ and is a stochastic variable, $l_{j}$ is the Impulse Range, and $\mathrm{I}_{j}$ is the Impulse of the agent (in Table 1). During the roaming state a friction is applied to the thrust for the simulation time step $t+\Delta t$,

$$
\tau_{j}^{t+\Delta t}=\left\{\begin{array}{lc}
\tau_{j}^{t} \phi_{j} & \text { if } \tau_{j}^{t} \phi_{j}>\tau_{j}^{\lim } \\
\tau_{j}^{\lim } & \text { otherwise }
\end{array} .\right.
$$

Where $\tau_{j}^{t}$ is the Thrust in the Thrust Limit $\tau_{j}^{\lim }$ and $\phi_{j}$ is the Friction applied to the force.

\section{Hiding and Fleeing (Earthquake or targeted by predator)}

Carnivore goes into the hiding state when earthquakes occur and Herbivore goes into the fleeing state when targeted by a predator:

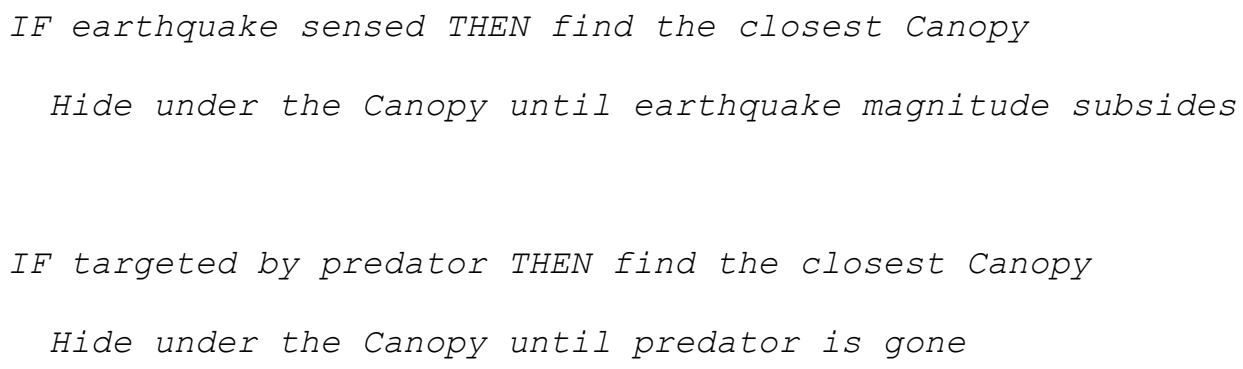




\section{Energy Gain and Loss}

Energy is gained via predation and food intake and energy loss occur during the movements. The use of energy depends on the efficiency of each species with the gain and loss,

$$
\lambda_{j}^{t+\Delta t}=\lambda_{j}^{t}+\kappa
$$

Where $\lambda_{j}^{t+\Delta t}$ is the energy of the agent $i$ at time $t+\Delta t$ and $\kappa$ defined in Table 2 is the amount of energy gained or lost. When Carnivore acquires Herbivore as food, the Flesh Index (Table 1) $K$ is added to the energy of the Carnivore. Herbivore acquires their energy from the Resource Index of the plants. The agent rests (stops activities) in the condition $\lambda_{j}^{t}<v_{j}$ where $v_{j}$ is the Energy Rest Threshold (Table 1).

\section{Table 2 - Energy gain and loss for both Herbivore and Carnivore}

States $\quad-\kappa$ Loss $\quad-\kappa$ Loss $\quad+\kappa$ Gain $\quad+\kappa$ Gain
(Herbivore)
(Carnivore)
(Herbivore)
(Carnivore)

\begin{tabular}{lcccc}
\hline ROAM & 0.0001 & 0.001 & - & - \\
HUNT & 0.0001 & 0.001 & - & - \\
FLEE & 0.001 & 0.001 & - & - \\
CHASE & - & 0.001 & - & - \\
SEARCH & 0.0001 & 0.001 & - & - \\
HIDE & 0.0001 & - & 0.1 & 0.01
\end{tabular}

\subsubsection{The Object-Oriented Implementation Details}

During the preparation for the exhibition, it was decided that Adobe Flash Action Script 3.0 (ObjectOrientation) should be used for the development for practical reasons (i.e., portability to the Web), however, the general mathematics algorithms can be applied to any object-oriented languages, such as a separate complex systems built for large-scale simulation in Java/C++ (Eugene Ch'ng, 2013), or a simplified version of plant distribution for virtual environments and visualization (Ch'ng, 2011). Details that are not covered in the Submodels section are covered here. Figure 8 shows an overview of the classes. Figure 9 and 10 illustrates the properties and methods of the class relationships between the agents and the environment. These important figures are included in the journal so that the Mixed Reality community can reproduce the work. 
The Main class acts as the controller for the entire system - setting up the user interface, initializing the agent and environment objects, updating, and managing and rendering the graphics Sprite layers associated with each agents (UILayer, SoilLayer, HerbivoreLayer, CarnivoreLayer, CanopyLayer, VegetableLayer, PoisonLayer). Note that the Sprite layers are to make sure that the rendering order is correct. For example, the SoilLayer should be the lowest layer (rendered first as a background) while the CanopyLayer should be at the top (rendered last).

The subsequent paragraphs explain the Environment and Agent classes in Figures 9 and 10 in more detail. For the purpose of more effectively visualizing the UML diagrams, the Flash event methods (Event, Keyboard, Mouse, Timer, etc.) are not shown in the diagrams.

\section{FIGURE 8 HERE}

The Environment class manages the connection with data input from the hardware sensors. Each variable in this list are linked to the hardware sensors - sunlight, temperature, humidity, $\mathrm{pH}$, and isTremor. The isTremor requires other related variables to work. The timeHandler() and updateLiveStream() function manages the isTremor variable. timeHandler() is updated every seconds and updateLiveStream() reads the sensors every $0.012 \mathrm{~ms}$. The pseudo code describes the process:

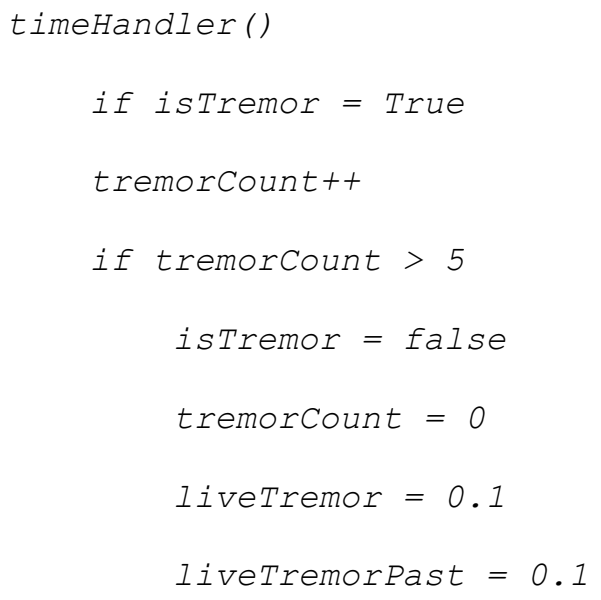

The three plant agents - Canopy, Poison, and Vegetable use the PlantInfo class for accessing the genotype parameters stored in an XML file. Each object derived from the classes senses the live stream from the abiotic sensors from the Environment object. The timeHandler() method manages the age increment of the plant and 
senescence (when age > maxAge). The update() method manages the rendering of the frames (Figure 9), calls the fitness() methods, and checks for scheduled reproduction.

\section{FIGURE 9 HERE}

The LifeProcesses class is used by all agents for measuring its adaptability to biotic and abiotic factors. All agents are made aware of other species via the Collection variables - plants, preys, predators. Each agent has a unique identification index and the agents identify their food target, competitors, and protection (Canopy) via these variables - competitorIndex, foodTarget, plantIndex, and coverIndex. The index of a particular target are obtained from the functions - ProximityTest() and FindPreyType(). The Carnivore and Herbivore class uses the AnimalInfo class to access their genotype via an XML file. These vagile agents are differentiated from the plant agents by the AnimalState, movement variables and methods, and the lesser number of abiotic factors that affect them.

\section{FIGURE 10 HERE}

\section{Discussions}

This section is a reflection of our artwork after having successfully collaborated in this interdisciplinary project, in having designed, implemented and deployed our exhibit in multiple venues, and interacted with participants representing a diverse range of demographics.

In this project, we explored a particular interest on how an artwork can better engage with audiences via a robust Mixed Reality interface, and as a positive consequence, investigated an innovative approach in converging the real and the virtual in a true, Mixed Reality system. The content of our artwork was Darwinian adaptation for survival. For this participatory art, we "mixed" reality by simulating virtual life which can be interacted with via actual physical human activity, converging the virtual and the physical in an innovative Mixed Reality system which we have formalized. We accomplished a truly mixed reality system by integrating real-time display, artificial life, ecological simulation, and real-time sensors, and physical props in a participatory artwork. Life itself and the shaping of life by nature's forces have always fascinated humankind. A mixed reality system requires such an aspect of work, our experience with the Shift-Life exhibit suggests that virtual life can lengthen viewer participation, fascinate audience, promote discussions, and enhance content sustainability. Our unique Shift-Life approach was the propitious provision by Darwin's Bicentenary Shift-Time Festival, and the eventful gathering of two faculties, allowing us to explore, and consequently experiment with such an artwork, which we believe extends the concept of Mixed Reality to another level - for what is true Mixed Reality, if one of the worlds, which lie on the one side of the interface, is lifeless? 
Traditionally, digital media artists tend to engage with their viewers either through a computer screen or projection and direct keyboard or mouse manipulation, or through the physical triggering of sensors to activate their work, for Shift-Life we were concerned with a more direct "relational" and participatory approach where viewers would both intuitively engage with the installation's everyday objects, and also with each other to more fully experience the piece as a Mixed Reality artwork. Our work is in contrast with contemporary artists, such as the Squid Soup collective and Jane Prophet, both of whom are digital media artists with an interest in computer games and play, as evident in their works which are similar to computer games such as Framsticks: $3 D$ simulation and evolution and MIT's Creatures. Squid Soup has done works which project light shapes of insectlike creatures into a box of sand where they could run over your hands, these having no AI programmed into them. TechnoSphere created (1995-2002) by Jane Prophet/Gordon Selley was an online virtual world for users to build creatures and release them into a real-time 3D simulation environment where they would interact. The program was re-launched as TechnoSphere III in 2012 with AI programming for simple evolution, the creatures responded as individuals to each other and the environment, they would chase or evade each other, eat, grow, mate and die. However, the creatures were completely immersed in a virtual world. In contrast, our artwork has a strong basis in Darwinian principles, brought forth through a Duchampian understanding of art, while Squid Soup, and Prophet's artworks were largely concerned with novelty and entertainment. The Duchampian understanding shifts from the aesthetics of art object, to the ideas held within that object, and that an art object needs a viewer to "complete" it through mental engagement and understanding, and such is our Mixed Reality artwork, an attempt at elucidating Darwin's thinking using an interactive interface. By transposing the "big idea" of Darwin into a participatory exhibit, we created a liminal space, blurring the perceived virtual and real states to the point where our viewers might suspend the belief that these life forms were artificial, and thus engage mentally with our work at a deeper level.

In our collaboration, in the first stage of development, there were questions on the adaptation and natural selection of our creatures which we hope to address further for future projects, these were questions on the evolution of our creatures and how we could appropriately represent evolution within a limited time-allowance for participatory exhibits, and in terms of the feasibility of project man-hours. The Shift-Life project has a limited time-span from funding to exhibition, and, due to constraints of time, we were unable to develop algorithms that simulate evolution (and for good reasons as participant viewing times were not long enough to witness evolution anyways, which requires significant generations of reproductions and mutations to occur, and within evolutionary timescales). Nevertheless, we believe that we have achieved important aspects of Darwin's observation in our simulation.

We have also acquired experience in Mixed Reality exhibits - the constant flow of visitors activating the sensors by hammering, pouring liquids etc., indicated that for longer sessions with more participants, we will 
need to design more robust methods for protecting the sensors and a more efficient way of dealing with the excess liquids collected underneath the display. Our future project should result in a more sophisticated Mixed Reality system, building from the foundational work described here. The new system should act as a platform for deeper thoughts on the understanding of interactive and participatory art practice. Our further exploration of both the conceptual and systemic aspect of Mixed Reality should yield further insights and techniques for the community interested in our work. Our creatures, programmed with simple rules have produced a multiplicity of behaviors during the exhibitions, some of which were surprising and unpredictable. This phenomenon also expresses the participatory art to a higher level - life is sustainable and therefore, the observation of our artwork is also sustainable. In the future, we hope to program and animate the creatures not only to rapidly evolve behavior in accordance with their volatile world, but to also have the creatures emerge unpredictable social patterns from the individually programmed behaviors, and thus bring the Darwinian principle to the full.

From observing members of the public engaging with the art installation it was pleasing to note that they needed no encouragement to do so, in that they found the implements playful and used them intuitively as intended. The simple creature aesthetics was therefore the right approach to delivering this sophisticated artificial life system to a non-science audience particularly where the simple 2D child-focused creatures and plants were concerned. On replaying the videos captured during the event, you can clearly hear the participants talking about the piece and their growing understanding of Darwinian adaptation for survival while also grasping the analogy to climate change affecting our actual world. They worked easily together in a relational way, often with complete strangers, to either nurture the creatures or disturb their environment. This is evident that they had experienced a meaningful encounter with the artwork on show and had obviously enjoyed this experience: "That's well good! Where did you get it from? ... you made it? WOW!"

Finally, we present our lead artist Dew Harrison's reflection as a summary conclusion to our article:

The collaboration with computer science and an animator allowed me to explore a means of engaging viewers in my artwork in an immediate and more fulfilling way. The aesthetics of the piece, the look and feel of it were important here where I was to elicit scientific principles to a nonscience audience. The idea was to make hard science and complex technical details disappear for the participants, to allow for Ihdeian “embodiment relations" (Ihde, 1990) to take place where the perception of technology "withdraws" into the body and the human experience through technology which is barely noticed by the users. Getting collaborators with the right skills was an important part of this project, each accomplishing their role in developing the installation in tune with my overall vision for the piece. I was delighted with the end product which chimed so well with my original idea. This has led me to re-consider my approach to practice, new works will now move away from screen-based projections and advance the inter-relationship between a virtual 
Duchampian world and the viewers' world through Mixed Reality interfaces and artificial life simulation.

\section{Acknowledgements}

Our thanks for funding and support go to, the Arts Council England, the Shrewsbury Museums Service, and the Shift-Time Festival organizers.

\section{References}

Azuma, R., Baillot, Y., Behringer, R., Feiner, S., Julier, S., \& MacIntyre, B. (2001). Recent advances in augmented reality. Computer Graphics and Applications, IEEE, 21(6), 34-47.

Azuma, R. T. (1997). A survey of augmented reality. Presence, 6(4), 355-385.

Barker, T. (2009). Process and (mixed) reality: A process philosophy for interaction in mixed reality environments. In Mixed and Augmented Reality-Arts, Media and Humanities, 2009. ISMAR-AMH 2009. IEEE International Symposium on (pp. 17-23). IEEE.

Breckling, B., Müller, F., Reuter, H., Hölker, F., \& Fränzle, O. (2005). Emergent properties in individual-based ecological models - introducing case studies in an ecosystem research context. Ecological Modelling, 186(4), 376-388.

Ch'ng, E. (2007). Modelling the Adaptability of Biological Systems. The Open Cybernetics and Systemics Journal, 1, 13-20.

Ch'ng, E. (2009). An Artificial Life-Based Vegetation Modelling Approach for Biodiversity Research. In R. Chiong (Ed.), Nature-Inspired informatics for Intelligent Applications and Knowledge Discovery: Implications in Business, Science and Engineering. (pp. 68-118). Hershey, PA.: IGI Global.

Ch'ng, E. (2011). Spatially Realistic Positioning of Plants for Virtual Environments: Simple Biotic and Abiotic Interaction for Populating Terrains. IEEE Computer Graphics and Applications, (99).

Ch'ng, E. (2013). Model resolution in complex systems simulation: Agent preferences, behavior, dynamics and n-tiered networks. SIMULATION, 89(5), 635-659.

Costanza, E., Kunz, A., \& Fjeld, M. (2009). Mixed reality: A survey. Springer.

Gillman, M., \& Hails, R. (1997). An introduction to ecological modelling: putting practice into theory. Blackwell Science.

Grasset, R., Woods, E., \& Billinghurst, M. (2007). Art and Mixed Reality: New Technology for Seamless Merging Between Virtual and Real.

Grimm, V., Berger, U., Bastiansen, F., Eliassen, S., Ginot, V., Giske, J., ... Huse, G. (2006). A standard protocol for describing individual-based and agent-based models. Ecological Modelling, 198(1), 115-126.

Grimm, V., \& Railsback, S. F. (2013). Individual-based modeling and ecology. Princeton university press.

Gwilt, I. (2009). Augmented reality and mobile art. In Handbook of multimedia for digital entertainment and arts (pp. 593-599). Springer.

Harrison, D., Ch'ng, E., Mount, S., \& Moore, S. (2009). Experiencing the Big Idea. In 8th International Conference on Digital Arts and Culture, DAC'09 12-15 December 2009. Irvine, California, USA.

Ihde, D. (1990). The Indiana Series in the Philosophy of Technology. Indiana University Press.

Langton, C. G. (1997). Artificial life: An overview. Mit Press.

Lombard, M., \& Ditton, T. (1997). At the heart of it all: The concept of presence. Journal of ComputerMediated Communication, 3(2), 0.

Luciani, A., Urma, D., Marlière, S., \& Chevrier, J. (2004). PRESENCE: the sense of believability of inaccessible worlds. Computers \& Graphics, 28(4), 509-517.

Maes, P. (1995). Artificial life meets entertainment: lifelike autonomous agents. Communications of the ACM, 38(11), 108-114.

Marshall, J. (2009). Creating illusion in computer aided performance. University of Nottingham.

Milgram, P., \& Kishino, F. (1994). A taxonomy of mixed reality visual displays. IEICE TRANSACTIONS on Information and Systems, 77(12), 1321-1329.

Misker, J. M. V, \& van der Ster, J. (2010). Authoring immersive mixed reality experiences. In The Engineering 
of Mixed Reality Systems (pp. 275-291). Springer.

Resnick, M. (1994). Turtles, termites, and traffic jams: Explorations in massively parallel microworlds. Mit Press.

Reynolds, C. W. (1987). Flocks, herds and schools: A distributed behavioral model. ACM Siggraph Computer Graphics, 21(4), 25-34.

SHAW, J., KENDERDINE, S., \& COOVER, R. (n.d.). Embodied Interfaces: Legible City and Distributed Legible City; conFiguring the CAVE; Web of Life.

Sims, K. (1994). Evolving virtual creatures. In Proceedings of the 21 st annual conference on Computer graphics and interactive techniques (pp. 15-22). ACM.

Whitelaw, M. (2004). Metacreation: art and artificial life. Mit Press. 